\title{
A Qualitative Study Exploring the Impact and Effects Following Hospital Discharge of COVID-19
}

\author{
Abigail Reay ${ }^{1}$, Avinash Aujayeb ${ }^{2, * \mathbb{D}}$, Catherine Dotchin ${ }^{2}$, Ellen Tullo ${ }^{3}$, John Steer ${ }^{2,3}$, Katherine Swainston ${ }^{4}$ \\ and Lorelle Dismore $3,5, *$
}

Citation: Reay, A.; Aujayeb, A.; Dotchin, C.; Tullo, E.; Steer, J.;

Swainston, K.; Dismore, L. A Qualitative Study Exploring the Impact and Effects Following Hospital Discharge of COVID-19. J. Respir. 2021, 1, 216-222. https:// doi.org/10.3390/jor1030020

Academic Editor: Cesar A. Moran

Received: 10 July 2021

Accepted: 27 August 2021

Published: 4 September 2021

Publisher's Note: MDPI stays neutral with regard to jurisdictional claims in published maps and institutional affiliations.

Copyright: (C) 2021 by the authors Licensee MDPI, Basel, Switzerland. This article is an open access article distributed under the terms and conditions of the Creative Commons Attribution (CC BY) license (https:/ / creativecommons.org/licenses/by/ $4.0 /)$.
1 British Psychological Society, Qualification in Health Psychology, St. Andrews House, Leicester LE1 7DR, UK; Abigail.reay2@gmail.com

2 Respiratory Department, Northumbria Healthcare NHS Foundation Trust, Northumbria Way, Cramlington NE23 6NZ, UK; Catherine.dotchin@nhct.nhs.uk (C.D.); john.steer@nhct.nhs.uk (J.S.)

3 Translational and Clinical Research Institute, Faculty of Medical Sciences, Newcastle University, Newcastle upon Tyne NE2 4HH, UK; ellen.tullo@nhct.nhs.uk

4 School of Social Sciences, Humanities \& Law, Campus Heart, Teesside University, Southfield Road, Middlesbrough TS1 3BX, UK; k.swainston@tees.ac.uk

5 Innovation, Research and Development, Northumbria Healthcare NHS Foundation Trust, North Tyneside Hospital, Rake Lane, North Shields NE29 8NH, UK

* Correspondence: avinash.aujayeb@nhct.nhs.uk (A.A.); Lorelle.dismore@northumbria-healthcare.nhs.uk or lorelle.dismore@nhct.nhs.uk (L.D.); Tel.: +44-0191-293-4087 (L.D.)

\begin{abstract}
Introduction: Research into the long-term effects of coronavirus disease 2019 (COVID-19) continues at an unprecedented pace. Many physical long-term symptoms of COVID-19 have been reported and include headache, fatigue, muscle pain and breathlessness, etc. Psychological effects are not dissimilar to survivors of SARS. There is limited qualitative research exploring the mental health impacts and experiences of hospitalized COVID-19 inpatients. Methods: A prospective qualitative study is planned to explore patient experiences post hospital discharge following a diagnosis of COVID-19. The research aims to gain an understanding of how COVID-19 affects quality of life (QoL) and functional abilities. Patients discharged from the hospital will be invited to take part in semi-structured interviews discussing their experiences of hospitalization and the impact of COVID19 on their QoL. Interviews will be conducted at three and six months following discharge from hospital. This study will provide important qualitative insight and may inform clinical interventions and commissioning decisions. Trial registration: The study has Research Ethics Committee (REC) and Health Research Authority (HRA) approvals obtained from Health and Care Research Wales (HCRW) [IRAS project ID 293196].
\end{abstract}

Keywords: COVID-19; Long COVID; QoL; qualitative; semi-structured; interviews

\section{Introduction}

The World Health Organization (WHO) declared Coronavirus 2019 (COVID-19), the multisystem disease caused by the severe acute respiratory syndrome coronavirus 2 (SARSCoV-2), as a pandemic on 11 March 2020 [1]. Commonly presenting symptoms are fever, cough, breathlessness and fatigue [2]. Individuals older than 60 years of age with comorbidities, such as cardiovascular disease, diabetes, hypertension and chronic obstructive pulmonary disease (COPD), are at the highest risk of severe COVID-19 and death [3]. At the time of writing, it is estimated that 185,125,237 people have had COVID-19, and 4,002,924 have died from COVID worldwide [4]. Approximately 20\% of those with COVID-19 are estimated to need hospitalization [1]. Of those admitted to hospital, depending on age, frailty and co-morbidities, mortality can be as high as $95 \%$. Approximately $17 \%$ require admission to high dependency or intensive care units and survival in these sub-groups can be as low as $28 \%$ [5]. The length of inpatient stay with COVID-19 may depend on higher levels of care and on severity of illness [6]. 
The constellation of persisting signs and symptoms (typically longer than 12 weeks), which can encompass headache, fatigue, muscle pain and breathlessness, has been termed 'Post COVID Syndrome' or 'Long COVID' [7]. Patients have described symptoms lasting weeks to months, with up to $87 \%$ of previously hospitalized patients being affected in some cohorts [8,9]. Fatigue, dyspnoea, joint pain and chest pain are commonly reported, and $40 \%$ of patients report a worsened quality of life (QoL) [9]. Those who have been discharged from intensive care may have significant long-lasting sequelae. Halpin et al. [10] reported that new illness-related fatigue was the most commonly reported symptom of patients discharged from the ICU following a COVID-19 diagnosis (72\%), followed by breathlessness ( $65.5 \%$ of ICU patients and $42.6 \%$ of ward patients) and psychological distress (46.9\% in ICU patients and $23.5 \%$ of ward patients). Their results also indicated a significant drop in quantitatively measured QoL (68.8\% of ICU patients and $45.6 \%$ in ward patients). Huang et al. [11] reported similar findings with approximately two thirds of patients reporting fatigue and muscle weakness, a quarter reporting sleep difficulty and a quarter reporting anxiety and depression six months after hospital discharge.

Research into the in-hospital experiences of patients with COVID-19 is limited. Inferences can be made from the 2003 severe acute respiratory syndrome (SARS) outbreak. Patients surviving SARS reported several psychiatric morbidities including persistent depression, anxiety, panic attacks, psychomotor excitement, psychotic symptoms, delirium and suicidal ideation $[12,13]$. The mental health status of patients whilst hospitalized with COVID-19 suffers: $45.9 \%$ had symptoms of depression, 38.8\% had anxiety and $54.1 \%$ had insomnia. Greater self-perceived illness severity was significantly associated with increased symptoms of low mood and anxiety [14]. Additionally, patients admitted to hospital with COVID-19, a potentially fatal disease, may experience fear resulting in increased anxiety [15]. Due to the risks of disease transmission, patients with COVID-19 often need to be isolated in rooms or wards within the hospital. In the United Kingdom (UK), this isolation has often been compounded by visiting restrictions; contact with family members or friends usually limited to telephone or video calls unless the patient was approaching end of life. Such isolation instills boredom, loneliness and irritability [16]. It is well documented that a lack of socialization can result in depression, anxiety and exacerbate physical health conditions [17]. R Boyes discloses that, following a five-week hospital stay, which included mechanical ventilation, he found living independently a struggle, and reported symptoms of insomnia, memory loss and a sense of abandonment from medical professionals [18]. While physical recovery is the primary aim during hospitalization, the psychological impact and quality of life of patients post discharge must thus not be overlooked.

Quantitative research can enable an objective understanding of patient experiences, however, qualitative methods enable a meaningful understanding of an individual's thoughts, emotions, attitudes and lived reality of their experiences. Thus, we have set out to explore patient experiences of being hospitalized with COVID-19 using qualitative research.

\section{Materials and Methods}

\subsection{Study Design}

The study is qualitative and phenomenological: patients surviving hospitalization for COVID-19 will be invited to take part in two semi-structured interviews (three and six months post-discharge from hospital). A qualitative design will ensure rich in-depth data are obtained regarding patient's experiences.

Aims and Objectives

- To understand the experiences of patients who have been hospitalized with COVID-19;

- To understand the impact of COVID-19 on patients' QoL;

- To understand longer-term impacts of COVID-19 on patients QoL to guide the development of appropriate interventions and services to meet the needs of patients. 


\subsection{Participants}

Inclusion criteria are:

- $\quad$ Patients aged $\geq 18$ years hospitalized with COVID-19 (polymerase chain reaction [PCR] swab positivity for SARS-CoV-2 and a clinical diagnosis of COVID-19 according to the admitting medical team;

- Discharged from hospital;

- Ability to provide informed consent;

- Willingness to take part in the interviews over the phone or by video call;

Exclusion criteria are:

- Inability to give informed consent.

Previous research into sample sizes in qualitative research has suggested gaining participants and interviews until the data analysis and transcription reaches saturation. Saturation has been found to come at different sample sizes depending on the research aims and objectives. It has been suggested that, if the qualitative research has specific research question and aims, little new information is generated after the twelfth interview. The research suggests that if the sample is more heterogenous, saturation may be researched with a larger sample [19]. Therefore, semi-structured interviews will be conducted until data saturation is reached. Patients will take part in one semi-structured interview conducted over the telephone in the comfort of their own home.

\subsubsection{Sampling Technique and Identification}

The clinical team will identify eligible participants and provide the research team with the participant's details. Following eligibility confirmation, a study pack containing an invitation letter and participant information sheet will the posted to potential participants. The researcher will telephone the potential participant within seven days to discuss the study in more detail and allow time to ask questions. For those potential participants requiring more time to consider participation, a follow-up telephone call will be scheduled for the following week. Additionally, patients can be identified prospectively by the clinical team. For those who are identified prospectively, the clinical team will have the option to provide the patient with a participation information sheet or the researcher will post a study pack; thereafter, participation will be followed up by the research team.

\subsubsection{Consent}

Participants have the option to complete witnessed consent or postal consent.

Witnessed consent: the researcher will read the statements on the consent form to the participant for their verbal agreement, with the participant, the researcher and a witness for the researcher present. The consent form will be signed by the researcher and the witness.

Postal consent: the researcher will post a consent form to the participant and a call will be scheduled in for the following week. The researcher will talk the patient through the consent form and the form will be signed by the patient. The researcher will then document the patient's name, and the date and time of the consent call. The patient will return the original completed consent form to be signed by a member of the research team. There will be a discrepancy in dates between the patient and researcher signatures.

\subsection{Study Interventions}

Following consent, the researcher will contact the patient to arrange a convenient time and date to conduct the telephone/video interview. The patient will be interviewed in the comfort of their own home, and the interviews will last no longer than one hour (See Appendix A for interview schedule). Verbal consent will be obtained prior the interviews to re-confirm that the patient is happy to be interviewed (See Figure 1). Interviews will be audio-recorded and destroyed once transcribed verbatim. Patients' demographic details will be obtained along with the virus strain and whether the patient has been vaccinated. 


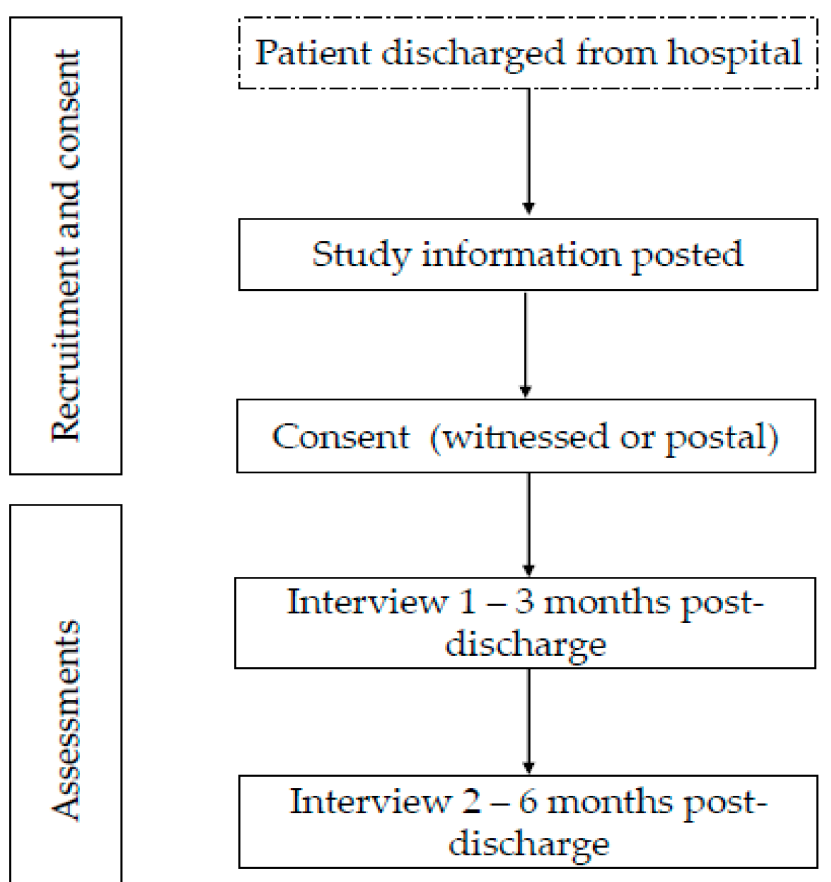

Figure 1. Recruitment and Data collection Pathway.

\subsection{Risk of Bias}

To avoid the risk of researcher bias, all interviews will be transcribed verbatim and will be reviewed and analyzed by more than one researcher. All themes and subthemes identified will be agreed upon by all researchers. All participants will be asked the same, open ended questions and the interviewer will not provide any information regarding the hospital treatment of the patient prior to the interview. To reduce recruitment bias, all participants will be asked to pick a convenient time for their interview, including evenings and weekends. Additionally, adults of all ages are able to take part in the research interview.

\subsection{Patient and Public Involvement}

Patients have been asked to comment on the acceptability of the research and give feedback on the Participant Information Sheet. A group will also be invited to review the results and discuss dissemination of study findings.

\subsection{Data Analysis}

Thematic Analysis will be used to analyze the transcripts. Thematic analysis is a form of qualitative research that aims to gather an understanding of a certain phenomenon from taking common themes from a larger body of data. It has been widely used in research regarding health and wellbeing to learn from the experiences of others [20]. Interviews will be read and re-read to ensure familiarization whilst documenting initial codes through identifying interesting features of the data; once codes are established, they will be collated into potential themes. The relevant quotations will be reviewed, and the final themes will be generated. To ensure inter-rated reliability, data will be analyzed by two researchers independently and interpretations of themes will be shared and discussed to agree upon key themes that have emerged from the data.

\subsection{Approvals}

Ethical approval has been granted by Health and Care Research Wales (HCRW) [IRAS project ID 293196] prior to data collection in a National Health Service (NHS) secondary care setting and Caldicott approval has been granted [C3713]. 
All patients will be assigned a pseudonym to protect their identity and maintain confidentiality. The study will exclude individuals who do not have capacity to consent. Should an individual lose consent during the 'active' period of this study, then the participant will be withdrawn at that point. Permission to retain data gathered before loss of capacity is included in the participant consent form. Participants will have the right to withdraw at any time without a reason and it will be explained that the data collected up to the point of withdrawal will not be used unless they give their consent.

\subsubsection{Data Handling and Storage}

For those participants who provide postal consent, the participants name will be kept only on the consent forms. The name will only be available to the research team and will not be used in any scientific reports. The data will not be shared with third parties. The consent forms will be stored in a lockable cabinet, separate from any results of the study. Electronic data will be stored on a password-protected trust computer server with data encryption and automated back-up.

\subsubsection{Adverse Events}

We envisage no risk to the patients; however, if new issues are identified during the study, the research team will notify the clinical team. Patients will provide consent for the researcher to notify the clinical team.

If any participant were to disclose risk to themselves or others, as well as possible safeguarding concerns, the relevant risk management procedure would be implemented. The participants will be made aware that their information and data will be kept anonymous; however, if they were to disclose any harm to themselves or others then information will be shared amongst professionals and the correct support offered to the participant.

\section{Results}

Results will be published in peer reviewed journals and presented at national conferences.

\section{Conclusions}

We hope that adding patients' voices via this study will increase our understanding of COVID-19 and its effects. Expected limitations are that this study might take several months to achieve recruitment and full analysis, that no formal result verification of the data is possible (as the participants have control over the content of the data) and that inferring causality might not be possible. Further discussion is beyond the scope of this protocol paper.

Author Contributions: All authors were involved in conceiving the study, C.D., E.T., A.A. and J.S. will identify eligible participants and L.D. will consent the participants into the study. A.R. and L.D. are responsible for conducted and transcribing the interviews. K.S. will be the third reviewer to reach consensus of the identified themes. All authors have contributed to writing the manuscript of the protocol for publication. All authors have read and agreed to the published version of the manuscript.

Funding: This research received no external funding.

Institutional Review Board Statement: The study was conducted according to the guidelines of the Declaration of Helsinki, and approved by an Ethics Committee.

Informed Consent Statement: Informed consent will be obtained from all subjects involved in the study.

Data Availability Statement: Due to maintaining confidentiality of the participants, the interview transcripts will not be made publicly available.

Acknowledgments: The authors would like to acknowledge the patients who will be taking the time to participate in this research and share their experiences.

Conflicts of Interest: The authors declare no conflict of interest. 


\section{Appendix A}

Table A1. Interview Questions.

\section{Interview Questions}

(1) Could you tell me about your experience of being on a hospital ward whilst having a diagnosis of COVID-19?

(2) How did you feel being admitted to hospital with a COVID-19 diagnosis?

(3) How long was your stay?

(4) Do you feel this was the correct length of stay/do you feel this should have been shorter/longer?

(5) Can you describe your treatment?

(6) What could have been done differently?

(7) How would you describe your overall experience?

(8) How would you describe your emotional wellbeing during your hospital stay?

(9) How satisfied overall are you with the care and treatment you received?

(10) How have you felt since your discharge from hospital?

(11) How do you feel your life has been impacted following discharge?

(12) How are you managing with your day to day activities?

(13) Are these any different to before you were admitted to hospital?

(14) What are your experiences of managing your self-care now?

(15) How has this changed compared to before your hospital stay?

(16) Have you had to make any changes regarding your personal hygiene?

(17) Have you experienced any long-term symptoms?

(18) Is there anything that you now struggle with that you didn't before?

(19) How have you felt overall in regards to the social restrictions of COVID-19 and how has this impacted on your life?

(20) How have you felt in terms of your emotional wellbeing since your discharge?

(21) Have you had to make any adaptations to your daily routine?

(22) Have you had any extra support/care packages put in place following your hospital stay?

(23) Is there anything you feel has specifically changed following your hospital stay?

(24) Is there else you would like to know about your quality of life following discharge?

\section{References}

1. World Health Organization. WHO Director-General's Opening Remarks at the Media Briefing on COVID-19-11 March 2020. Available online: https:/ / www.who.int/director-general/speeches/detail/who-director-general-s-opening-remarks-at-themedia-briefing-on-covid-19---11-march-2020 (accessed on 31 July 2021).

2. Zhou, Z.; Zhang, M.; Wang, Y.; Zheng, F.; Huang, Y.; Huang, K.; Yu, Q.; Cai, C.; Chen, D.; Tian, Y.; et al. Clinical characteristics of older and younger patients infected with SARS-CoV-2. Aging (Albany NY) 2020, 12, 11296-11305. [CrossRef] [PubMed]

3. Shahid, Z.; Kalayanamitra, R.; McClafferty, B.; Kepko, D.; Ramgobin, D.; Patel, R.; Aggarwal, C.S.; Vunnam, R.; Sahu, N.; Bhatt, D.; et al. COVID-19 and older adults: What we know. J. Am. Geriatr. Soc. 2020, 68, 926-929. [CrossRef] [PubMed]

4. COVID-19 Map-Johns Hopkins Coronavirus Resource Center. Available online: https://coronavirus.jhu.edu/map.html (accessed on 31 July 2021).

5. Docherty, A.B.; Harrison, E.M.; Green, C.A.; Hardwick, H.E.; Pius, R.; Norman, L.; Holden, K.A.; Read, J.M.; Dondelinger, F.; Carson, G.; et al. Features of 20133 UK patients in hospital with covid-19 using the ISARIC WHO Clinical Characterisation Protocol: Prospective observational cohort study. BMJ 2020, 369, m1985. [CrossRef] [PubMed]

6. Rees, E.M.; Nightingale, E.S.; Jafari, Y.; Waterlow, N.R.; Clifford, S.; Pearson, C.A.B.; CMMID Working Group; Jombart, T.; Procter, S.R.; Knight, G.M. COVID-19 length of hospital stay: A systematic review and data synthesis. BMC Med. 2020, 18, 1-22. [CrossRef] [PubMed]

7. Garner, P. For 7 Weeks I Have Been Through a Roller Coaster of Ill Health, Extreme Emotions, and Utter Exhaustion; BMJ Opinion: London, UK, 2020.

8. Mahase, E. Covid-19: What do we know about “long covid"? BMJ 2020, 370, m2815. [CrossRef] [PubMed]

9. Carfi, A.; Bernabei, R.; Landi, F. For the Gemelli Against COVID-19 Post-Acute Care Study Group Persistent Symptoms in Patients After Acute COVID-19. JAMA 2020, 324, 603-605. [CrossRef] [PubMed]

10. Halpin, S.J.; McIvor, C.; Whyatt, G.; Adams, A.; Harvey, O.; McLean, L.; Walshaw, C.; Kemp, S.; Corrado, J.; Singh, R.; et al. Postdischarge symptoms and rehabilitation needs in survivors of COVID-19 infection: A cross-sectional evaluation. J. Med. Virol. 2020, 93, 1013-1022. [CrossRef] [PubMed]

11. Huang, C.; Huang, L.; Wang, Y.; Li, X.; Ren, L.; Gu, X.; Kang, L.; Guo, L.; Liu, M.; Zhou, X.; et al. 6-month consequences of COVID-19 in patients discharged from hospital: A cohort study. Lancet 2021, 397, 220-232. [CrossRef] 
12. Liu, T.B.; Chen, X.Y.; Miao, G.D.; Zhang, L.; Zhang, Q.; Cheung, T. Recommendations on diagnostic criteria and prevention of SARS-related mental disorders. J. Clin. Psychol. Med. 2003, 13, 188-191.

13. Maunder, R.; Hunter, J.; Vincent, L.; Bennett, J.; Peladeau, N.; Leszcz, M.; Sadavoy, J.; Verhaeghe, L.M.; Steinberg, R.; Mazzulli, T. The immediate psychological and occupational impact of the 2003 SARS outbreak in a teaching hospital. Can. Med. Assoc. J. 2003, $168,1245-1251$.

14. Hu, Y.; Chen, Y.; Zheng, Y.; You, C.; Tan, J.; Hu, L.; Zhang, Z.; Ding, L. Factors related to mental health of inpatients with COVID-19 in Wuhan, China. Brain Behav. Immun. 2020, 89, 587-593. [CrossRef] [PubMed]

15. Xiang, Y.T.; Yang, Y.; Li, W.; Zhang, L.; Zhang, Q.; Cheung, T.; Ng, C.H. Timely mental health care for the 2019 novel coronavirus outbreak is urgently needed. Lancet Psychiatry 2020, 7, 228-229. [CrossRef]

16. Brooks, S.K.; Webster, R.; E Smith, L.; Woodland, L.; Wessely, S.; Greenberg, N.; Rubin, G.J. The psychological impact of quarantine and how to reduce it: Rapid review of the evidence. Lancet 2020, 395, 912-920. [CrossRef]

17. Windle, K.; Francis, J.; Coomber, C. Preventing Loneliness and Social Isolation: Interventions and Outcomes; Social Care Institute for Excellence: London, UK, 2011.

18. Boyes, R. Patient perspective: Roger Boyes. BMJ 2020, 369, m1813. [CrossRef] [PubMed]

19. Vasileiou, K.; Barnett, J.; Thorpe, S.; Young, T. Characterising and justifying sample size sufficiency in interview-based studies: Systematic analysis of qualitative health research over a 15-year period. BMC Med. Res. Methodol. 2018, 18, 1-8. [CrossRef]

20. Braun, V.; Clarke, V. What can "thematic analysis" offer health and wellbeing researchers? Int. J. Qual. Stud. Health Well-Being 2014, 9, 26152. [CrossRef] [PubMed] 